\title{
Total Synthesis of Endangered Frog Alkaloid 'Epibatidine', A Potent Analgesic of the Future
}

\author{
Dhatrak, N.R. and M.G. Kulkarni \\ Department of Chemistry, University of Pune, Pune 411007, India
}

Article history

Received: 05-11-2014

Revised: 28-11-2014

Accepted: 31-12-2014

Corresponding Author:

Dhatrak, N.R.

Department of Chemistry,

University of Pune, India

Email: ajit@chem.unipune.ac.in

\begin{abstract}
Epibatidine' is a nicotinic alkaloid', was first discovered in the skin of neotropical poisonous frog, 'Epipedobates tricolor' found in Ecuador. John Daly in 1974 first isolated a trace quantity of this alkaloid by scarifying several frogs. With the evolution of NMR techniques the structure of this alkaloid was reported in 1992. An year after, Nobel Laureate Prof. E.J. Corey, devised first total synthesis for this alkaloid. Since then this molecule is constantly attracting the attention of Epibatidine is an agonist at nicotinic acetylcholine receptor. Pharmacologically it is 200 to 300 times stronger than morphine and do not cause any addition. The frog species 'Epipedobates tricolor' is now been declared as endangered and therefore there is special need for easy syntheses to get the alkaloid for biological evaluations and to save these frogs from extinction. We have developed a Wittig Claisen based protocol for the total synthesis of this drug molecule which would help in investigation rest pharmacological activities of this drug. Structure of Epibatidine Molecule and the frog species from which it was isolated.
\end{abstract}

Keywords: Epibatidine, Wittig-Claisen Protocol

\section{Introduction}

Epibatidine; a pyridine alkaloid, is an agonist at nicotinic acetylcholine receptor. Pharmacologically it is a potent analgesic alkaloid. It was first discovered in the skin of neotropical poisonous frog, 'Epipedobatestricolour' found in Ecuador (Fig. 1). John Daly (NIH, Bethesda, Md) in 1974 first isolated one milligram of this alkaloid by scarifying several frogs (Daly et al., 1994). This species now been declared endangered and therefore there is special need for easy syntheses to get the alkaloid for more biological studies and to save these frogs from extinction.

With evolution of NMR techniques the structure of this alkaloid was reported in 1992 (Daly et al., 1994). Year latter Nobel Laureate Prof. E.J. Corey devised first synthetic method for this alkaloid (Corey et al., 1993). Since then this molecule is constantly attracting the attention of Organic chemists and biochemists because of its strong analgesic activities. It is 200 to 300 times stronger than morphine and do not cause any addition. This molecule now a day being envisaged an analgesic of the future (Fig. 2).

\section{Materials and Methods}

The required 6-chlronicotinic acid was purchased from Sigma-Aldrich and Merck. Other chemicals and reagents were purchased from Spectrochem. THF was dried over active sodium metal wire and distilled from dark blue colored benzophenone radical. All the NMRs were recorded using Varian Mercury 300MHz Instrument using $\mathrm{CDCl}_{3}$ as solvent except the target Epibatidine molecule which was a dihydrochloride salt and was recorded in $\mathrm{D}_{2} \mathrm{O}$ as solvent. IR and mass analysis was done using Shimadzu and instruments respectively.

\section{Results and Discussion}

Witting-Claisen protocol is a well-developed methodology in our laboratory (Fig. 3). We have successfully extended this methodology for the synthesis of several natural products. Above said protocol has been successfully applied to the synthesis of Epibatidine. Allyl vinyl ether synthesized from 6-chloronicotinaldehyde was rearranged under Claisen rearrangement conditions to construct required carbon skeleton.

6-chloronicotinic acid which is commercially available in market was reduced to get corresponding alcohol-2. This alcohol was oxidized using pyridiniumchlorochromate to get aldehyde-3. Aldehyde-3 was then subjected to Wittig olefination using allyloxymethylenetriphenylphosphranechloride (Kulkarni et al., 2010a; 2010b) salt to get corresponding allyl-vinyl-ether-4 as an inseparable mixture of cis and trans isomers. 


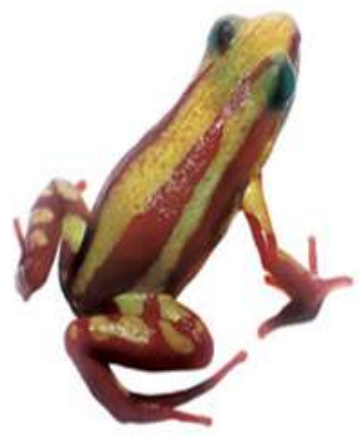

Fig 1. Epipedobatestricolor

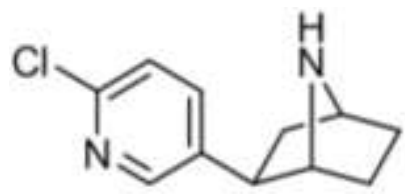

Fig. 2. Structure of epibatidine molecule
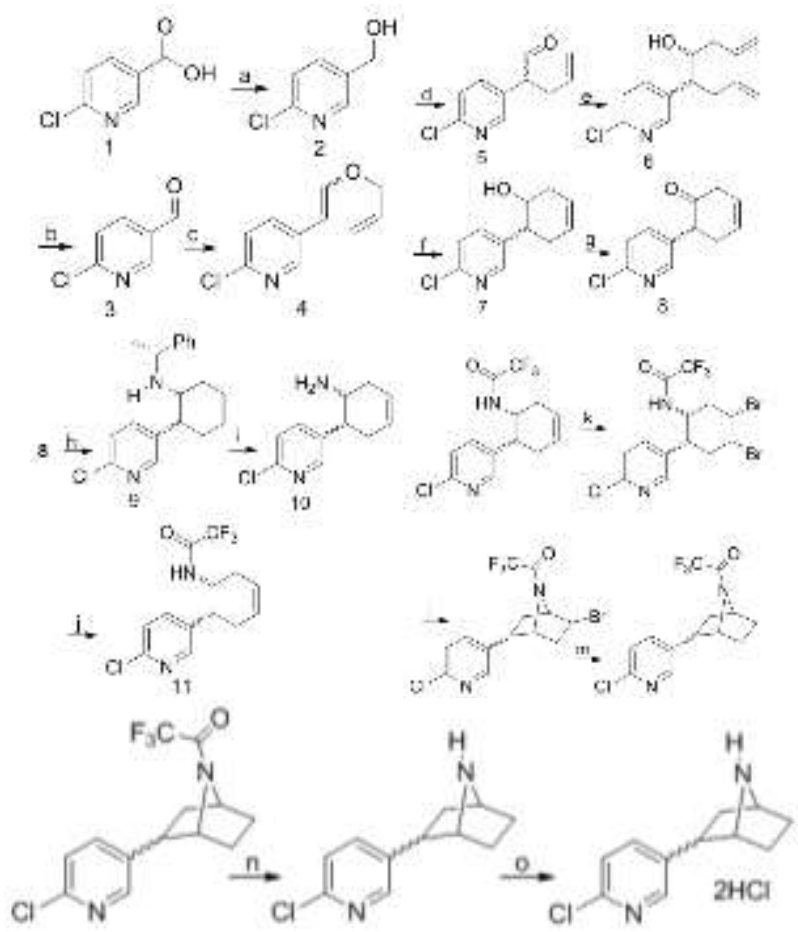

Fig. 3. (a) LAH, THF, $\mathrm{O}^{0} \mathrm{C}$ to RT (b) $\mathrm{PCC}, \mathrm{DCM}, 4 \mathrm{~A}^{\circ} \mathrm{MS}, \mathrm{RT}$ (c) $\mathrm{CH}_{2}=\mathrm{CHCH}_{2} \mathrm{OCH}_{2} \mathrm{P}^{+} \mathrm{Ph}_{3} \mathrm{Cl}^{-}, \mathrm{t}-\mathrm{BuONa}, \mathrm{THF}, 0^{\circ} \mathrm{C}$ (d) $160^{\circ} \mathrm{C}$, Neat, $10 \mathrm{~min}$ (e) $\mathrm{In}^{\circ}$, allylbromide, aq THF $\left(30 \% \mathrm{H}_{2} \mathrm{O}\right), \mathrm{RT}, 8 \mathrm{hrs}$ (f) Grubb's Catalyst II ${ }^{\text {nd }}$ gen 5 mol\%, DCM, 24 hrs. (g) PDC, DCM, RT, 3hrs (h) R-()- $\alpha$-methylbenzylamine, anhydrous $\mathrm{MgSO}_{4}$, dry metnanol, $\mathrm{NaBH}_{3} \mathrm{CN}$ (i) $\mathrm{Pd} / \mathrm{C}, \mathrm{H}_{2}$ atmosphere, DCM, (j) Trifluoroacetic anhydride, Et $3 \mathrm{~N}, \mathrm{DCM} 20^{\circ} \mathrm{C}(\mathrm{k}) \mathrm{Br}_{2}$, Et4N ${ }^{+} \mathrm{B}^{\mathrm{r}}-$, DCM, $\left.\left.-78^{\circ} \mathrm{C}, 1\right) \mathrm{KOtBu}, \mathrm{THF},-78^{\circ} \mathrm{C}, \mathrm{m}\right)$ $\mathrm{Bu}_{3} \mathrm{SnH}, \mathrm{AIBN}, \mathrm{PhH}$, reflux (n) $\mathrm{NaOMe}, \mathrm{MeOH}, 25^{\circ} \mathrm{C}$, (o) $\mathrm{HCl}(\mathrm{g}), 0^{\circ} \mathrm{C}, \mathrm{DCM}$
The allyl-vinyl ether-4 so obtained was subjected to Claisen rearrangement in xylene which shows several thermolytic products. This problem was resolved using neat Claisen conditions. This amendment improved the yield of aldehyde-5up to $92 \%$.

The aldehyde 5 was directly used for further process without any purification and was processed using indium metal mediated Barbierallylation. The product diene-6 was purified using column chromatography. Ring closure metathesisof this product diene- 6 gave a cyclohexenol derivative-7 with $90 \%$ yield. The cyclohexene moiety obtained so was oxidized to get ketone- 8 . The cyclohexanone moiety 8 was subjected to reductiveamination using optically pure $\alpha$ methylbenzylamine with an intension to get separable distereomers.

The racemic amine 9 was then deprotected to corresponding amine 10. This amine was again protected using trifluoroaceticanhydride (Corey et al., 1993). Synthesized and further synthesis and the olefin function were brominated. The dibromo derivative undergoes ready cyclization on treatment of a base to give desired azabicycoheptane carbon skeleton (Corey et al., 1993). Reduction of bromo group and deprotection of amine resulted in the target molecule 'Epibatidine'.

Taking in consideration the relative instability of the epibatidine, it was converted into its dihydrochloride salt. $\mathrm{HCl}$ gas was bubbled in DCM solution of the Epibatidine to get Epibatidinedihydrochloride.

The crystalline material obtained was washed with hexane several times and was analyzed using NMR spectroscopy in $\mathrm{D}_{2} \mathrm{O}$. NMR characteristics of this compound were found identical to previous reports. The method discussed herein gives racemic form of Epibatidine. Further modification of this method in order to achieve optically pure form ofEpibatidine is in progress.

\section{Conclusion}

In conclusion we have developed a novel synthetic method for Epibatidine using Wittig-Claisen protocol.

\section{Acknowledgement}

Authors are thankful for generous funding received from University Grants Commission (UGC).

\section{Author's Contributions}

All authors equally contributed in this work.

\section{Ethics}

This article is original and contains unpublished material. The corresponding author confirms that all of 
the other authors have read and approved the manuscript and no ethical issues involved.

\section{References}

Corey, E.J., T.P. Loh, S. AchyuthaRao, D.C. Daley and S. Sarshar, 1993. Stereocontrolled total synthesis of (+)and (-)-epibatidine. J. Org. Chem., 58: 5600-5602. DOI: 10.1021/jo00073a013

Daly, J.W., F. Gusovsky, C.W. Myers, M. YotsuYamashita and T. Yasumoto, 1994. First occurrence of tetrodotoxin in a dendrobatid frog (Colostethus inguinalis), with further reports for the bufonid genus Atelopus. Toxicon, 32: 279285.

DOI: 10.1016/0041-0101(94)90081-7
Kulkarni, M.G., S.W. Chavhan, M.P. Desai, Y.B. Shaikh and Dnyaneshwar D. Gaikwad et al., 2010a. A short and efficient synthesis of furo[2,3-b]indoles. Tetrahedron Lett., 51: 4494-4496. DOI: 10.1016/j.tetlet.2010.06.068

Kulkarni, M.G., Y.B. Shaikh, A.S. Borhade, A.P. Dhondge and S.W. Chavhan et al., 2010b. The efficient synthesis of (3R,3aS,6aR)hexahydrofuro[2,3-b]furan-3-ol and its isomers. Tetrahedron: Asymmetry, 21: 2394-2398. 